\title{
Cytotoxic effect of antioxidants found in food from plant origin on human osteosarcoma U2OS Cells
}

\author{
Efeito citotóxico de antioxidantes de alimentos de origem vegetal em células de osteossarcoma \\ humano U2OS
}

\author{
Marisa Nicolai, Nuno Almeida, Patrícia Rijo, João Guilherme Costa, Nuno Saraiva, Ana Sofia Fernandes \\ CBIOS - Universidade Lusófona's Research Center for Biosciences and Health Technologies, Campo Grande, 376, 1749- \\ 024, Lisboa, Portugal \\ Email: marisa.nicolai@ulusofona.pt
}

\begin{abstract}
Plants are a major source of natural products with antioxidant properties. Some of these phytochemicals have been used in effort to prevent and treat several human diseases, including cancer. Several studies support the effects of dietary antioxidants as chemopreventive agents, while other reports suggest potential pharmacological anticancer activity. However, the available studies were conducted under different experimental conditions, precluding a comparative analysis of the anticancer properties of the compounds.

Although osteosarcoma has a low overall incidence, it is the third most common cancer in adolescence. These cancers are generally locally aggressive and tend to produce early systemic metastases, justifying the search for novel therapeutic approaches.

This work assessed the cytotoxic profile of ten antioxidants from food from plant origin on human osteosarcoma cells (U2OS): catechin, kaempferol, quercetin, resveratrol, gallic acid, ferulic acid, ascorbic acid, melatonin, lycopene, and $\beta$-carotene. Cell viability was determined using the crystal violet assay $(24 \mathrm{~h})$. $\beta$-carotene and gallic acid considerably reduced cell viability, with IC50 values of $18.8 \mu \mathrm{M}$ and $184.5 \mu \mathrm{M}$, respectively. The remaining compounds did not markedly reduce cell viability. These results might contribute to the rational and evidence-based selection of natural antioxidants to be included in anticancer strategies and to further explore possible food-inspired drugs.
\end{abstract}

Keywords: Dietary antioxidants, cancer, osteosarcoma, U2OS, cytotoxicity

\section{Resumo}

As plantas são uma das principais fontes de produtos naturais com propriedades antioxidantes. Alguns destes fitoquímicos têm atraído atenção devido ao seu papel na prevenção e tratamento de doenças. Vários estudos assinalam estes antioxidantes como agentes quimiopreventivos, enquanto outros sugerem potenciais propriedades farmacológicas antitumorais. Contudo, os estudos disponíveis foram realizados em diferentes condições, impossibilitando uma análise comparativa.

O osteossarcoma tem uma baixa incidência global, mas é o terceiro tipo de cancro mais comum na adolescência. Este tipo de cancro é localmente agressivo e tende a produzir metástases precocemente, justificando a procura de novas abordagens terapêuticas.

Este trabalho avaliou o perfil citotóxico de dez antioxidantes de alimentos vegetais em células de osteossarcoma humano (U2OS): catequina, kaempferol, quercetina, resveratrol, ácido gálico, ácido ferúlico, ácido ascórbico, melatonina, licopeno e $\beta$-caroteno. A viabilidade celular foi determinada usando o ensaio de violeta de cristal (24 h). O $\beta$-caroteno e o ácido gálico reduziram consideravelmente a viabilidade celular, com valores de IC 50 de 18,8 e 184,5 $\mu \mathrm{M}$, respetivamente. Os restantes compostos não reduziram significativamente a viabilidade celular.

Palavras chave: antioxidantes da dieta, cancro, osteossarcoma, U2OS, citotoxicidade 


\section{Introduction}

According to WHO (2018) cancer, the rapid creation of abnormal cells that grow beyond their normal margins, and which can then invade contiguous parts of the body and spread to any other organs, is the second foremost cause of global death and is responsible for 9.6 million estimated deaths in 2018. One-third of deaths from malignant tumors are related with dietary and lifestyle habits, such as high body mass index, low fruit and vegetable intake, lack of physical activity, alcohol and tobacco use $(1,2)$

Osteosarcoma, a rare sarcoma that has histological origin in osteoid production in association with malignant mesenchymal cells, is one of the most common primary malignant tumors of bone and is prevalent in children and adolescents. This type of tumor can arise sporadically, with few cases associated with known inherited defects in cell cycle regulation. About $70 \%$ of tumor specimens demonstrated a chromosomal anomaly (3). Oxidative stress is a key component to associate environmental toxicity with the multistage carcinogenic process, characterized by the generation of reactive oxygen species (ROS) in response to endogenous and exogenous stimuli. In order to counterbalance ROS-mediated damages, the organism has endogenous antioxidant defense systems that include antioxidant enzymes (e.g., superoxide dismutase, catalase, glutathione peroxidase, and glutathione reductase) and non-enzymatic antioxidants (e.g., carotenoids, hormones, polyphenols, and vitamins) that have the ability to quench ROS (4).

Many epidemiological studies suggest that some types of food may contribute to reduce the risk of cancer. Food from plant origin, including vegetables and fruits, are in this category being rich in antioxidants, have been associated in the literature to anticancer properties $(5,6)$. Although most studies focus on the effects of dietary antioxidants as chemopreventive agents, other reports suggest potential pharmacological anticancer properties. However, the published studies were carried out under different experimental conditions, precluding a comparative analysis of the anticancer properties of the compounds. Therefore, the aim of this study was to evaluate the cytotoxic effect of ten different dietary antioxidants under the same experimental conditions. The effects of catechin, kaempferol, quercetin, resveratrol, gallic acid, ferulic acid, ascorbic acid, melatonin, lycopene, and $\beta$-carotene, commonly found in plant-based foods, were evaluated on an in vitro model of human osteosarcoma - the U2OS cell line.

\section{Introdução}

De acordo com a OMS (2018), o cancro, a rápida proliferação de células anormais que crescem além de suas margens normais, e que podem invadir partes contíguas do corpo e espalhar-se para outros órgãos, é a segunda causa de morte global e é responsável por cerca de 9,6 milhões de mortes, valores estimados em 2018. Um terço das perdas causadas por tumores malignos estão relacionadas com hábitos alimentares e estilo de vida, onde se destacam o alto índice de massa corporal, a reduzida ingestão de frutas e hortaliças, a ausência de atividade física, o uso de álcool e tabaco $(1,2)$.

O osteossarcoma, neoplasia rara que pode ser identificada histologicamente através da observação de osteóides em associação com células mesenquimais malignas, é um dos tumores malignos primários ósseos mais comuns e é prevalente em crianças e adolescentes. Este tipo de tumor pode surgir esporadicamente, existindo poucos casos associados a anomalias hereditárias relacionadas com a regulação do ciclo celular. Contudo, cerca de $70 \%$ de amostras tumorais demonstraram anomalia cromossómica (3).

O stress oxidativo é uma componente chave que associa a toxicidade ambiental com o processo de cancerigénese multietapas, o qual se caracteriza pela geração de espécies reativas de oxigénio (ERO) em resposta a estímulos endógenos e exógenos. A fim de compensar os danos mediados pelas ERO, o organismo possui sistemas endógenos de defesa antioxidante que incluem enzimas antioxidantes (por exemplo, superóxido dismutase, catalase, glutationa peroxidase e glutationa redutase) e antioxidantes não-enzimáticos (por exemplo, carotenóides, hormonas, polifenóis e vitaminas) que têm a capacidade de mitigar a atividade das ERO (4). Um grande número de estudos epidemiológicos sugere que alguns tipos de alimentos podem contribuir para a redução do risco de cancro. Nesta categoria estão os alimentos vegetais, incluindo hortaliças e frutas, que são ricos em antioxidantes e têm sido associados na literatura com propriedades antitumorais $(5,6)$. Embora a maioria dos estudos saliente os efeitos dos antioxidantes fitoquímicos alimentares como agentes quimiopreventivos, outros sugerem potenciais propriedades farmacológicas anticancerígenas. Entretanto, os estudos disponíveis foram realizados em diferentes condições experimentais, impossibilitando uma análise comparativa das propriedades anticancerígenas dos compostos. Deste modo, o objetivo deste estudo foi avaliar o efeito citotóxico de dez diferentes antioxidantes dietéticos nas mesmas condições experimentais. Os efeitos de catequina, kaempferol, quercetina, resveratrol, ácido 


\section{Materials and methods}

\section{Chemicals}

Dulbecco's Modified Eagle's Medium (DMEM), fetal bovine serum (FBS), penicillin-streptomycin solution, crystal violet, kaempferol, quercetin, gallic acid, ferulic acid, ascorbic acid, and melatonin were purchased from Sigma-Aldrich (St Louis, MO, USA). Dimethylsulfoxide (DMSO), ethanol and acetic acid were purchased from Merck (Darmstadt, Germany). Catechin and $\beta$-carotene were purchased from Cayman (Ann Arbor, MI, USA). Resveratrol and lycopene were purchased from Gonmisol (Barcelona, Spain).

\section{Cell culture}

The human osteosarcoma cell line U2OS was maintained in DMEM medium supplemented with $10 \%$ FBS, $100 \mathrm{U} / \mathrm{mL}$ penicillin, and $0.1 \mathrm{mg} / \mathrm{mL}$ streptomycin according to Saraiva and collaborators previous works (7). The cultures were maintained at $37^{\circ} \mathrm{C}$, under a humidified atmosphere containing $5 \% \mathrm{CO}_{2}$ in air.

\section{Crystal violet staining assay}

Cell viability was evaluated by the crystal violet (CV) staining assay. Approximately 3000 cells in $190 \mu \mathrm{L}$ of culture medium per well were seeded in 96-well plates and incubated for 24 hours. Cell were then exposed to antioxidant compounds catechin $(1-86 \mu \mathrm{M})$, kaempferol $(1-437 \mu \mathrm{M})$, quercetin $(1-10 \mu \mathrm{M})$, resveratrol $(5-100 \mu \mathrm{M})$, gallic acid $(10-2000 \mu \mathrm{M})$, ferulic acid $(1-1000 \mu \mathrm{M})$, ascorbic acid $(10-2000 \mu \mathrm{M})$, melatonin (1-645 $\mu \mathrm{M})$, lycopene $(0.5-3.9 \mu \mathrm{M})$ and $\beta$ - carotene (1$56 \mu \mathrm{M}$ ), for 24 hours. The CV assay was carried out according to a protocol previously described by Fernandes and colleagues (8). DMSO solutions at $0.5 \%$ and $5 \%(\mathrm{v} / \mathrm{v})$ were used as negative and positive control, respectively. A minimum of two and a maximum of four independent experiments were performed, each comprised of four replicate cultures. The half maximal inhibitory concentration $\left(\mathrm{IC}_{50}\right)$ was calculated using GraphPad Prism Statistical Software (version 7).

\section{Results and discussion}

The anticancer properties of dietary derived compounds had been previously explored using osteosarcoma models. Osteosarcoma models, however, differences in the experimental conditions and models used limit any comparative analyses. In this work, we compared the impact of ten common dietary antioxidants in the cell gálico, ácido ferúlico, ácido ascórbico, melatonina, licopeno e $\beta$-caroteno, comumente encontrados em alimentos vegetais, foram avaliados num modelo in vitro de osteossarcoma humano - a linha celular U2OS.

\section{Materiais e métodos}

\section{Reagentes}

Meio de cultura Dulbecco's Modified Eagle's Medium (DMEM), soro bovino fetal (FBS), solução de penicilina-estreptomicina, tripsina, violeta de cristal (CV), kaempferol, quercetina, ácido gálico, ácido ferúlico, ácido ascórbico e melatonina foram adquiridos à Sigma-Aldrich (St Louis, MO, EUA). Dimetilsulfóxido (DMSO), etanol e ácido acético foram comprados à Merck (Darmstadt, Alemanha). Catequina e $\beta$-caroteno foram adquiridos à Cayman (Ann Arbor, MI, EUA). Resveratrol e licopeno foram adquiridos à Gonmisol (Barcelona, Espanha).

\section{Cultura celular}

A linha celular de osteossarcoma humano U2OS foi mantida no meio DMEM suplementado com $10 \%$ de FBS, $100 \mathrm{U} / \mathrm{mL}$ de penicilina e $0,1 \mathrm{mg} / \mathrm{mL}$ de estreptomicina de acordo com trabalhos anteriores de Saraiva e colaboradores (7). As culturas foram mantidas a $37^{\circ} \mathrm{C}$, sob atmosfera húmida, contendo $5 \%$ de $\mathrm{CO}_{2}$.

\section{Ensaio de coloração com violeta de cristal}

A viabilidade celular foi avaliada através do ensaio de coloração com cristal violeta $(\mathrm{CV})$. Adicionaram-se a aproximadamente 3000 células U2OS por cada $190 \mu \mathrm{L}$ de meio de cultura, por poço. As células foram semeadas em microplacas de 96 poços e incubadas por 24 horas. Após este período, as células foram então expostas aos compostos antioxidantes: catequina (1-86 $\mu \mathrm{M})$, kaempferol $(1-437 \mu \mathrm{M})$, quercetina $(1-10 \mu \mathrm{M})$, resveratrol (5-100 $\mu \mathrm{M})$, ácido gálico $(10-2000 \mu \mathrm{M})$, ácido ferúlico (1-1000 $\mu \mathrm{M})$, ácido ascórbico $(10-2000 \mu \mathrm{M})$, melatonina $(1-645 \mu \mathrm{M})$, licopeno $(0,5-3,9 \mu \mathrm{M})$ e $\beta$-caroteno (1-56 $\mu \mathrm{M})$, durante 24 horas. O ensaio CV foi realizado de acordo com o protocolo descrito previamente por Fernandes e colaboradores (8). DMSO 0,5\% e $5 \%$ $(\mathrm{v} / \mathrm{v})$ foram usados como controle negativo e positivo, respetivamente. Dois a quatro ensaios independentes foram realizados e cada um incluiu quatro replicados. A concentração inibitória 50 ( $\left.\mathrm{IC}_{50}\right)$ foi calculada usando o GraphPad Prism Statistical Software (versão 7). 
viability of a human cell line of osteosarcoma, using the same experimental protocol. Globally, a concentration-dependent decrease in cell viability was observed for most of the antioxidants studied (Figure 1). Most antioxidants (catechin, kaempferol, quercetin, resveratrol, ferulic acid, and ascorbic acid) led to cell viabilities above $60 \%$ in the concentrations tested.

To the best of our knowledge, there are no previous studies addressing the impact of isolated catechin in the viability of osteosarcoma cells. Huang and colleagues reported that kaempferol decreased the cell viability of osteosarcoma cells in a concentration dependent manner (0-200 $\mu \mathrm{M}, 24$ hours of incubation) (9). In our work, the decreases in cell viability were less pronounced. A previous report found that quercetin $(0-120 \mu \mathrm{M})$ induced a reduction in cell viability in the U2OS cell line following 48 hours of incubation in a concentration dependent manner (10). Although due to solubility constraints we have only tested lower concentrations of quercetin, we have also observed a trend towards a concentration-dependent reduction in cell viability. The distinct concentrations and period of exposure tested may justify the differences between our data and the previously published results. Similarly, resveratrol (0$100 \mu \mathrm{M})$ inhibited cell proliferation and induced apoptosis in four different osteosarcoma cell lines (HOS, MG-63, Saos-2, and U2OS) (11). The decreases in cell viability were more pronounced than ours, but longer periods of incubation ( 3 and 7 days) were used. Regarding ferulic (12) and ascorbic (13) acids, our decreases in cell viability are in line with those previously reported for similar concentrations and incubation period in other osteosarcoma cell lines.

In our study, no cytotoxic effects were found for lycopene under the experimental conditions used. The very limited solubility of this compound precluded the study of higher concentrations. Previously, Wakshlag and colleagues reported that lycopene decreased the proliferation of 3 canine osteosarcoma cell lines (D17, OS 2.4 and HMPOS). However, such effect was only found with longer incubation periods (14).

Previous studies on the effect of melatonin in osteosarcoma cell lines show conflicting results $(15,16)$. Interestingly, U2OS cells exposed to melatonin showed an increase in cell viability (Figure 1H), reaching 182.9 $\%$ for the highest concentration studied $(645 \mu \mathrm{M})$, suggesting a possible pro-proliferative effect of this compound.

Our results show that catechin, kaempferol, quercetin, resveratrol, ferulic acid, ascorbic acid, melatonin, and lycopene did not induce a pronounced reduction in U2OS cell viability under our experimental conditions. However, for a more complete screening of their anti-

\section{Resultados e discussão}

As propriedades antitumorais de compostos habitualmente encontrados em alimentos de origem vegetal foram anteriormente estudados em modelos de osteossarcoma. Os resultados disponíveis foram realizados em diferentes modelos e condições experimentais, impossibilitando uma análise comparativa das propriedades antitumorais desses compostos. De modo a contribuir para o preenchimento desta lacuna, descrevemos o impacto de dez antioxidantes alimentares comuns na viabilidade celular de uma linha celular de osteossarcoma humana, usando sempre a mesma metodologia experimental. De um modo geral, foi observada uma diminuição na viabilidade celular dependente da concentração, para a maioria dos antioxidantes utilizados (Figura 1). A maioria dos antioxidantes (catequina, kaempferol, quercetina, resveratrol, ácido ferúlico e ácido ascórbico) mantiveram a viabilidade celular acima de $60 \%$, nas concentrações testadas.

De acordo com o nosso conhecimento, não existem estudos prévios que abordem o impacto da catequina na viabilidade de células de osteossarcoma. Huang e colaboradores reportaram que o kaempferol diminuiu a viabilidade de células de osteossarcoma de forma dependente da concentração $(0-200 \mu \mathrm{M}, 24$ horas de incubação) (9). Neste trabalho, as reduções na viabilidade celular foram menos pronunciadas. Um trabalho anterior mostrou que a quercetina $(0-120 \mu \mathrm{M})$ induziu uma redução na viabilidade celular da linha celular U2OS, após 48 h de incubação, de modo dependente da concentração (10). Contudo, devido a restrições de solubilidade, nós testámos apenas concentrações mais baixas de quercetina, também observámos uma tendência para uma redução na viabilidade celular dependente da concentração. As concentrações e o período de incubação diferentes testados podem justificar as diferenças entre nossos resultados e os resultados publicados anteriormente. Da mesma forma, o resveratrol $(0-100 \mu \mathrm{M})$ inibiu a proliferação celular e induziu a apoptose em 4 diferentes linhas celulares de osteossarcoma (HOS, MG-63, Saos-2 e U2OS) (11). As reduções na viabilidade celular foram mais pronunciadas do que as nossas, mas foram usados períodos de incubação mais longos (3 e 7 dias). Relativamente aos ácidos ferúlico (12) e ascórbico (13), as reduções na viabilidade celular observadas por nós estão de acordo com as relatadas anteriormente para concentrações e período de incubação semelhantes em outras linhas celulares.

Neste estudo, não foram encontrados efeitos citotóxicos para o licopeno, nas condições experimentais utilizadas. A solubilidade muito limitada deste composto 

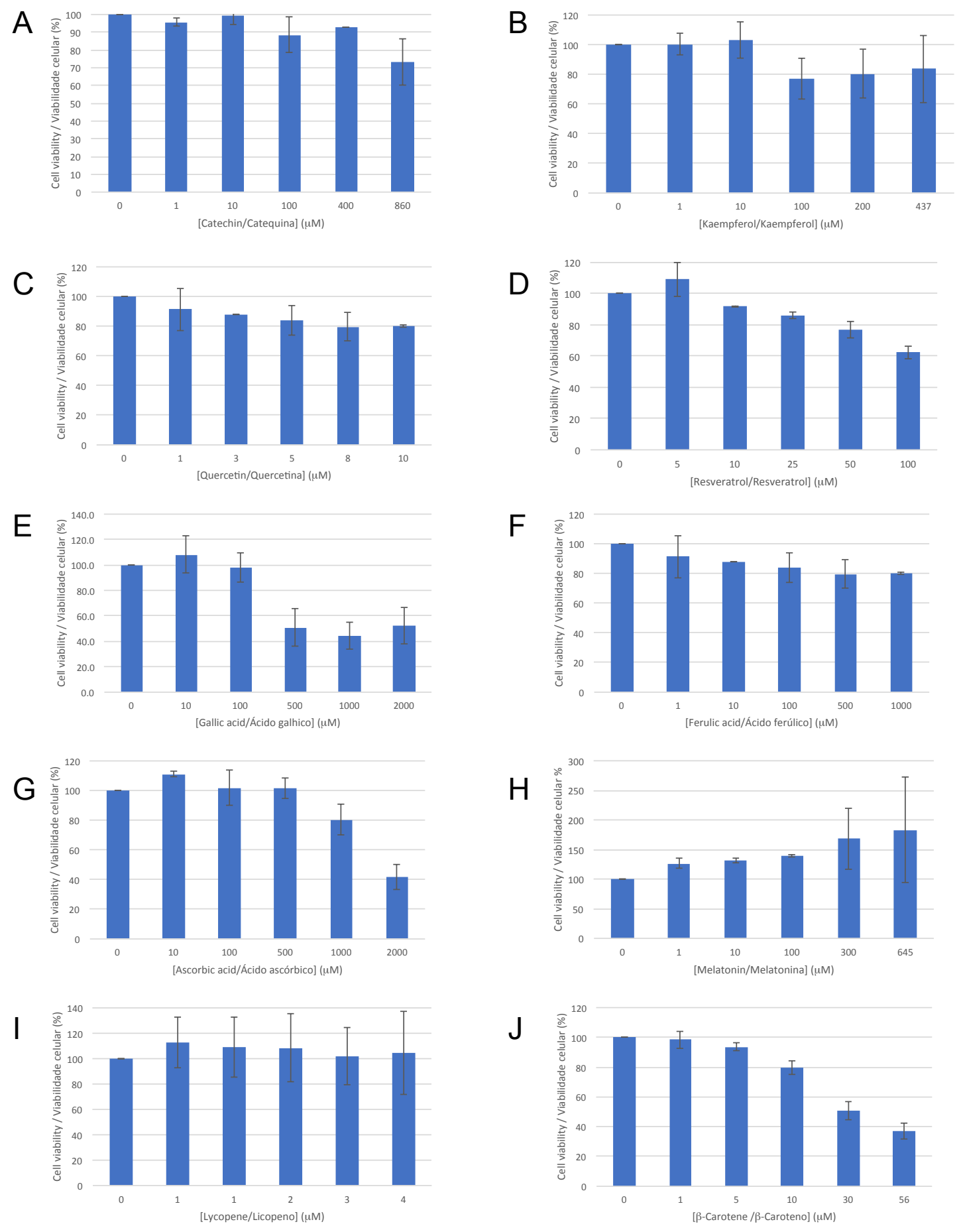

Figure / Figura 1 - Viability of U2OS cells after exposure to different antioxidants (A-catechin, Bkaempferol, C-quercetin, D-resveratrol, E-gallic acid, F-ferulic acid, G-ascorbic acid, H-melatonin, I-lycopene, $\mathrm{J}-\beta$-carotene) for 24 hours, assessed by the crystal violet assay. Values are expressed as means \pm SD from 2-4 independent experiments/ Viabilidade de células U2OS após incubação com diferentes antioxidantes (A-catequina, B-kaempferol, C-quercetina, D-resveratrol, E-ácido gálico, Fácido ferúlico, ácido G-ascórbico, H-melatonina, I-licopeno, J- $\beta$-caroteno), durante 24 horas, determinado através do método de violeta de cristal. Os valores são expressos como média \pm DP, com 2-4 ensaios experimentais independentes 
cancer properties, their cytotoxicity should be studied in additional cell models, representative of other cancer types. In addition, more prolonged incubation periods with the compounds should also be studied to allow the detection of possible mechanisms of delayed cell death. For gallic acid and $\beta$-carotene, a considerable reduction in the viability of osteosarcoma cells was observed, in the range of concentrations tested and for the 24 hours incubation period (Figure 1E and G). Therefore, it was possible to calculate the $\mathrm{IC}_{50}$ values of gallic acid and carotene, which were $184.5 \mu \mathrm{M}$ and $18.8 \mu \mathrm{M}$, respectively.

Our results for gallic acid are in the same order of magnitude, but less pronounced than those previously reported for this compound in osteosarcoma cell lines (U2OS and MNNG-HOS) for a 24 -incubation period $(17,18)$.

In our study, $\beta$-carotene was the dietary antioxidant with greater impact in reducing osteosarcoma cells' viability. Although carotenoids have been shown to modulate the hallmarks of cancer, there are no previous studies focused on the effects of $\beta$-carotene on osteosarcoma cells. According to the anticancer mechanisms described for $\beta$-carotene in other cell models, the observed decrease in cell viability can be ascribed to cell cycle arrest or apoptosis induction mechanisms (19).

The high-grade rate of metastases associated to osteosarcoma press the scientific community toward the discovery of new approaches to tackle this cancer (13). The results obtained in this work allow the choice of adequate concentrations to be used in subsequent studies addressing the impact of these compounds on cell proliferation, migration, and invasion, which are critical events for the formation of metastases.

\section{Conclusion}

$\beta$-carotene and gallic acid considerably reduced U2OS cell viability, with $\mathrm{IC}_{50}$ values of $18.8 \mu \mathrm{M}$ and 184.5 $\mu \mathrm{M}$, respectively. The remaining compounds did not markedly reduce cell viability.

These results might contribute to the rational and evidence-based selection of antioxidants to be included in dietary-based anticancer strategies and to further explore possible food-inspired drugs.

\section{Acknowledgments}

This work was financially supported by Fundação para a Ciência e a Tecnologia (FCT, Portugal), through pro- impossibilitou o estudo de concentrações mais elevadas. Anteriormente, Wakshlag e colaboradores relataram que o licopeno diminuiu a proliferação de 3 linhas celulares de osteossarcoma canino (D17, OS 2.4 e HMPOS). No entanto, esse efeito só foi encontrado com períodos de incubação mais longos (14).

Estudos anteriores sobre o efeito da melatonina nas linhas celulares de osteossarcoma mostram resultados contraditórios $(15,16)$. Curiosamente, as células U2OS expostas à melatonina mostraram um aumento na viabilidade celular (Figura $1 \mathrm{H}$ ), atingindo $182,9 \%$ para a concentração mais elevada estudada $(645 \mu \mathrm{M})$, sugerindo um possível efeito pró-proliferativo deste composto. Os nossos resultados mostram que a catequina, o kaempferol, a quercetina, o resveratrol, o ácido ferúlico, o ácido ascórbico, a melatonina e o licopeno não induziram uma redução significativa na viabilidade celular das células U2OS nas condições experimentais utilizadas. Contudo, para uma melhor avaliação das suas propriedades antitumorais, a sua citotoxicidade deve ser estudada em modelos celulares adicionais, representativos de outros tipos de cancro. Além disso, períodos de incubação mais prolongados com os compostos também deverão ser testados para permitir a deteção de possíveis mecanismos de morte celular tardia.

Para o ácido gálico e o $\beta$-caroteno, foi observada uma redução considerável na viabilidade das células de osteossarcoma, para o intervalo de concentrações testadas e para o período de incubação de 24 horas (Figura 1E e $\mathrm{G})$. Deste modo, foi possível calcular os valores de $\mathrm{IC}_{50}$ do ácido gálico e do $\beta$-caroteno, os quais foram 184,5 $\mu \mathrm{M}$ e $18,8 \mu \mathrm{M}$, respetivamente.

Os resultados obtidos para o ácido gálico são da mesma ordem de grandeza, mas menos pronunciados do que os previamente descritos para este composto em linhas celulares de osteossarcoma (U2OS e MNNG-HOS) para um período de incubação de 24 horas $(17,18)$.

Neste estudo, o $\beta$-caroteno foi o antioxidante com maior impacto na redução da viabilidade das células de osteossarcoma. Embora os carotenóides tenham vindo a demonstrar modular as características do cancro, não existem estudos prévios focados nos efeitos do $\beta$-caroteno em células de osteossarcoma. De acordo com os mecanismos antitumorais descritos para o $\beta$-caroteno em outros modelos celulares, a redução observada na viabilidade celular pode ser atribuída a mecanismos de paragem no ciclo celular ou indução de apoptose (19).

A elevada taxa de metástases associada ao osteossarcoma pressiona a comunidade científica para a descoberta de novas abordagens para o combate deste cancro (13). Os resultados obtidos neste trabalho permitem a escolha de concentrações adequadas a serem utilizadas em 
ject UID/DTP/04567/2016 (general funding to CBIOS and research grant attributed to Nuno Almeida).

\section{Conflict of interests}

The author declares that there is no personal or financial relationship that can be understood as presenting a potential conflict of interest. estudos subsequentes que abordarão o impacto destes compostos na proliferação, migração e invasão celular, os quais são eventos críticos para a formação de metástases.

\section{Conclusão}

O $\beta$-caroteno e o ácido gálico reduziram consideravelmente a viabilidade das células U2OS, com valores de $\mathrm{IC}_{50}$ de $18,8 \mu \mathrm{M}$ e $184,5 \mu \mathrm{M}$, respetivamente. Para os restantes compostos não houve uma acentuada redução da viabilidade celular.

Estes resultados contribuem para uma seleção racional e suportada na evidência de antioxidantes a serem incluídos em estratégias antitumorais baseadas na dieta e a explorar para o desenvolvimento de novos fármacos inspirados nos alimentos.

\section{Agradecimentos}

Este trabalho foi financiado através da Fundação para a Ciência e a Tecnologia (FCT, Portugal), através do projeto UID / DTP / 04567/2016 (financiamento geral CBIOS e bolsa de investigação atribuída a Nuno Almeida).

\section{Conflito de interesses}

O autor declara não existir qualquer relação pessoal ou financeira que possa ser entendida como potencial conflito de interesses. 


\section{References/Referências}

1. World Health Organization. Cancer. Retrieved October 4, 2018, from https://www.who.int/cancer/en/.

2. Siegel RL, Miller KD, Jemal A. Cancer statistics. CA Cancer J Clin 2019;69(1):7-34.

3. Misaghi A, Goldin A, Awad M, Kulidjian AA. Osteosarcoma: a comprehensive review. Sicot-J 2018; 4:12.

4. Fernandes AS, Castro M, Guerreiro Oliveira N. Oxidative stress and antioxidant defences - a pedagogical review. J Biomed Biopharm Res 2018; $8(1): 97-108$.

5. Kapinova A, Kubatka P, Golubnitschaja O, Kello M, Zubor P, Solar P, Pec M. Dietary phytochemicals in breast cancer research: anticancer effects and potential utility for effective chemoprevention. Environ Health Prev Med 2018; 23(1):36.

6. Shankar E, Kanwal R, Candamo M, Gupta S. Dietary phytochemicals as epigenetic modifiers in cancer: Promise and challenges. Semin Cancer Biol 2016; 40-41:82-99.

7. Saraiva N, Prole DL, Carrara G, Johnson BF, Taylor CW, Parsons M, Smith GL. hGAAP promotes cell adhesion and migration via the stimulation of store-operated Ca2+ entry and calpain 2. J Cell Biol 2013; 202(4):699-713.

8. Fernandes AS, Gaspar J, Cabral MF, Rueff J, Castro M, Batinic-Haberle I, Costa J, Oliveira NG. Protective role of ortho-substituted Mn(III) N-alkylpyridylporphyrins against the oxidative injury induced by tert-butylhydroperoxide. Free Radic Res 2010; 44(4):430-40.

9. Huang WW, Chiu YJ, Fan MJ, Lu HF, Yeh HF, Li KH, Chen PY, Chung JC, Yang JS. Kaempferol induced apoptosis via endoplasmic reticulum stress and mitochondria-dependent pathway in human osteosarcoma U-2 OS cells. Mol Nutr Food Res 2010; 54(11):1585-95.

10. Xie X, Yin J, Jia Q, Wang J, Zou C, Brewer KJ, Colombo C, Wang Y, Huang G, Shen J. Quercetin induces apoptosis in the methotrexate-resistant osteosarcoma cell line U2-OS/MTX300 via mitochondrial dysfunction and dephosphorylation of Akt. Oncol Rep 2011; 26(3):687-93.

11. Li Y, Bäckesjö CM, Haldosén LA, Lindgren U. Resveratrol inhibits proliferation and promotes apoptosis of osteosarcoma cells. Eur J Pharmacol 2009; 609(1-3):13-8.

12. Wang, T, Gong, X, Jiang R, Li H, Du W, Kuang G. Ferulic acid inhibits proliferation and promotes apoptosis via blockage of PI3K/Akt pathway in osteosarcoma cell. Am J Transl Res 2016; 8(2):968-80.

13. Valenti MT, Zanatta M, Donatelli L, Viviano G, Cavallini C, Scupoli MT, Dalle Carbonare L. Ascorbic acid induces either differentiation or apoptosis in MG-63 osteosarcoma lineage. Anticancer Res 2014; 34(4):1617-28.

14. Wakshlag JJ, Balkman CE. Effects of lycopene on proliferation and death of canine osteosarcoma cells. Am J Vet Res 2010; 71(11):1362-70.

15. Panzer A, Lottering ML, Bianchi P, Glencross DK, Stark JH, Seegers JC. Melatonin has no effect on the growth, morphology or cell cycle of human breast cancer (MCF-7), cervical cancer (HeLa), osteosarcoma (MG-63) or lymphoblastoid (TK6) cells. Cancer Lett 1998; 122(1-2):17-23.

16. Cheng Y, Cai L, Jiang P, Wang J, Gao C, Feng H, Wang C, Pan H, Yang Y. SIRT1 inhibition by melatonin exerts antitumor activity in human osteosarcoma cells. Eur J Pharmacol 2013; 715(1-3):219-29.

17. Liang C, Zhang X, Li H, Tao Y, Tao L, Yang Z, Zhou X, Shi Z, Tao H. Gallic Acid Induces the Apoptosis of Human Osteosarcoma Cells In Vitro and In Vivo via the Regulation of Mitogen-Activated Protein Kinase Pathways. Cancer Biother Radiopharm 2012; 27(10):701-10.

18. Liao CL, Lai KC, Huang AC, Yang JS, Lin JJ, Wu SH, Wood WG, Lin JG, Chung JC. Gallic acid inhibits migration and invasion in human osteosarcoma U-2 OS cells through suppressing the matrix metalloproteinase-2/-9, protein kinase B (PKB) and PKC signaling pathways. Food Chem Toxicol 2012; 50(5): $1734-40$.

19. Niranjana R, Gayathri R, Nimish Mol S, Sugawara T, Hirata T, Miyashita K, Ganesan P. Carotenoids modulate the hallmarks of cancer cells. J Funct Foods $2015 ; 18: 968-85$. 